\title{
ERROR OF THE TWO-STEP BDF FOR THE INCOMPRESSIBLE NAVIER-STOKES PROBLEM
}

\author{
ETIENNE EMMRICH ${ }^{1}$
}

\begin{abstract}
The incompressible Navier-Stokes problem is discretized in time by the two-step backward differentiation formula. Error estimates are proved under feasible assumptions on the regularity of the exact solution avoiding hardly fulfillable compatibility conditions. Whereas the time-weighted velocity error is of optimal second order, the time-weighted error in the pressure is of first order. Suboptimal estimates are shown for a linearisation. The results cover both the two- and three-dimensional case.
\end{abstract}

Mathematics Subject Classification. 35Q30, 65M12, 76D05.

Received: October 7, 2003.

\section{INTRODUCTION}

In comparison with the spatial approximation of the incompressible Navier-Stokes problem, only a small number of articles is concerned with a strict mathematical substantiation of time discretisation methods. A main problem in deriving higher-order error estimates is the requirement of higher regularity of the exact solution. This is equivalent to compatibility conditions on the problem's data that lead - due to the divergencefree constraint - to a virtually uncheckable and often violated over-determined Neumann problem for the initial pressure. So it seems to be inappropriate to assume higher regularity for proving higher-order error estimates. However, A- or G-stable methods can take advantage of parabolic smoothing properties that allow to derive non-smooth data error estimates.

Smoothing properties are also at hand for the Navier-Stokes solution. In [5], optimal second-order smoothing error estimates have been proven for the Crank-Nicolson scheme under feasible regularity assumptions. The fractional-step- $\theta$-scheme has been considered in [7], projection methods in [8].

In this paper, we shall consider the two-step backward differentiation formula (BDF) with constant time steps, which is known to be of formally second order and zero- as well as strongly A- and G-stable, for the Navier-Stokes problem in its pressure-free variational formulation. A linearized variant in which the convective term $\left(u^{n} \cdot \nabla\right) u^{n}$ is replaced by $\left(\left(2 u^{n-1}-u^{n-2}\right) \cdot \nabla\right) u^{n}$, where $u^{n}$ is the approximate velocity at time $t_{n}$, has been firstly studied in [4]. The optimal second-order error estimate for the velocity in the $1^{\infty}\left(\mathrm{L}^{2}\right)-\mathrm{and}^{2}\left(\mathrm{H}_{0}^{1}\right)$-norm given there relies upon higher regularity that leads to the above-mentioned over-determined Neumann problem. A similar estimate has been also postulated in [1]. Recently, sub-optimal error estimates for the velocity of

\footnotetext{
Keywords and phrases. Incompressible Navier-Stokes equation, time discretisation, backward differentiation formula, error estimate, parabolic smoothing.

1 Institut für Mathematik, TU Berlin, Straße des 17. Juni 136, 10623 Berlin, Germany. e-mail: emmrich@math.tu-berlin.de
} 
order $1 / 4$ in the $1^{\infty}\left(\mathrm{H}_{0}^{1}\right)$-norm have been proven in [6] under feasible regularity assumptions for solenoidal initial data in $\mathrm{H}_{0}^{1}$. This result applies to the two-dimensional case with autonomous right-hand side.

Error estimates for the original nonlinear approximation have not been considered in the literature so far. Moreover, the pressure approximation and its error have also not been studied. Solvability, stability of the discrete problem, and convergence of a prolongated, time continuous approximate solution towards a weak solution have been proven in [2] for the original nonlinear approximation and its linearized variant.

Here, we shall derive optimal error estimates for the nonlinear and sub-optimal estimates for the linearized approximation: The velocity error, measured in the natural $1^{\infty}\left(\mathrm{L}^{2}\right)$ - and $\mathrm{l}^{2}\left(\mathrm{H}_{0}^{1}\right)$-norm, is firstly shown to be of first order. Afterwards, we prove - via a duality trick - a second-order estimate for the time-weighted velocity error to the nonlinear approximation. For the linearized method, only order $3 / 2$ can be obtained. We also derive estimates for the time-weighted pressure error in the $1^{\infty}\left(\mathrm{L}^{2} / \mathbb{R}\right)$-norm, namely of first order for the nonlinear and of order $1 / 2$ for the linearized approximation. The order reduction in the pressure approximation is due to the difference between the dual spaces of $\mathrm{H}_{0}^{1}$ and its solenoidal subspace and the Babuška-Brezzi condition. The results apply to the two- and three-dimensional Navier-Stokes problem with time-dependent right-hand side.

We have also tried to focus on appearing time step restrictions. So it turns out that the first-order estimate for the linearized variant holds without any restriction on the step size whereas the result for the nonlinear approximation requires sufficiently small step sizes depending strongly on the Reynolds number Re. For a detailed quantification of the constants appearing (especially its dependence on Re), we refer to $[2,3]$.

\section{Continuous And Time DiscRete PROBlem}

We consider the Navier-Stokes equations describing the flow of an incompressible, homogeneous, viscous fluid at constant temperature in a bounded domain $\Omega \subset \mathbb{R}^{d}(d=\operatorname{dim} \Omega \in\{2,3\})$ with smooth boundary $\partial \Omega$,

$$
\begin{gathered}
u_{t}-\nu \Delta u+(u \cdot \nabla) u+\nabla p=f, \quad \nabla \cdot u=0 \quad \text { in } \Omega \times(0, T), \\
u=0 \quad \text { on } \partial \Omega \times(0, T), \quad u(\cdot, 0)=u_{0} \quad \text { in } \Omega,
\end{gathered}
$$

where $(0, T)$ is the time interval under consideration, $\nu=1 / \operatorname{Re}>0, u=u(x, t)$ is the $d$-dimensional velocity with initial velocity $u_{0}=u_{0}(x), p=p(x, t)$ is the pressure, and $f=f(x, t)$ is an outer force per unit mass. For the variational formulation, let us introduce the solenoidal Hilbert spaces $H:=\left\{v \in \mathrm{L}^{2}(\Omega)^{d}: \nabla \cdot v=0, \gamma v=0\right\}$ ( $\gamma$ denotes the trace operator in normal direction, $c f .[9]$ for more details) with the natural $\mathrm{L}^{2}$-norm $|\cdot|$ and inner product $(\cdot, \cdot)$ and $V:=\left\{v \in \mathrm{H}_{0}^{1}(\Omega)^{d}: \nabla \cdot v=0\right\}$ with norm $\|v\|:=|\nabla v|$ and inner product $((u, v)):=(\nabla u, \nabla v)$. By $\mathrm{L}^{2}, \mathrm{H}^{m}(m \in \mathbb{Z})$, and $\mathrm{H}_{0}^{1}$, we denote the usual Lebesgue and Sobolev spaces. Note that $V, H$ and the dual $V^{*}$ form a Gelfand triple. The dual pairing is denoted by $\langle\cdot, \cdot\rangle$, the dual norm in $V^{*}$ by $\|\cdot\|_{*}$. We consider

Problem (P). For given $u_{0} \in H$ and $f \in \mathrm{L}^{2}\left(0, T ; V^{*}\right)$, find $u \in \mathrm{L}^{2}(0, T ; V)$ such that for all $v \in V$

$$
\frac{\mathrm{d}}{\mathrm{d} t}(u(t), v)+\nu((u(t), v))+b(u(t), u(t), v)=\langle f(t), v\rangle
$$

holds in $(0, T)$ in the distributional sense with $u(0)=u_{0}$.

The nonlinearity is incorporated by the trilinear form $b(u, v, w):=((u \cdot \nabla) v, w)$. $\operatorname{By~}^{p}(S ; X)(p \in[1, \infty])$ for some time interval $S$ and a Banach space $X$, we denote the usual space of Bochner integrable abstract functions with its natural norm $\|\cdot\|_{\mathrm{L}^{p}(S ; X)}$.

Problem $(\mathrm{P})$ possesses at least one solution $u \in \mathrm{L}^{2}(0, T ; V) \cap \mathrm{L}^{\infty}(0, T ; H)$ with the distributional time derivative $u^{\prime} \in \mathrm{L}^{4 / 3}\left(0, T ; V^{*}\right)$. As $u$ is then almost everywhere equal to a continuous function with values in $V^{*}$ $\left(u \in \mathcal{C}\left([0, T] ; V^{*}\right)\right)$, the initial condition makes sense. In the two-dimensional case, the solution is unique, $c f$. [9]. If $u_{0} \in V, f \in \mathrm{L}^{\infty}(0, T ; H)$, and $\partial \Omega \in \mathcal{C}^{2}$, a unique strong solution $u \in \mathcal{C}([0, T] ; V)$ exists in the two-dimensional case for arbitrary $T$, but in the three-dimensional case only up to a possibly rather small time $T$, $c f$. [10]. 
In the sequel, let $A: V \rightarrow V^{*}$ be the energetic extension of the Stokes operator defined via $\langle A u, v\rangle:=$ $((u, v))$ for $u, v \in V$, which is linear, bounded, symmetric, and strongly positive. It follows $\|g\|_{*}=\left\|A^{-1} g\right\|=$ $\left\langle g, A^{-1} g\right\rangle^{1 / 2}$ for $g \in V^{*}$. It is further known that $A$ restricted to $\mathcal{D}(A):=\mathrm{H}^{2}(\Omega)^{d} \cap V \subset H$ (Friedrichs extension) is an isomorphism onto $H$. Due to Cattabriga's inequality, $|A \cdot|$ is equivalent to the $\mathrm{H}^{2}(\Omega)^{d}$-norm on $\mathcal{D}(A)$.

Theorem 1.1. Let $\partial \Omega$ be sufficiently smooth and let $u_{0} \in \mathcal{D}(A), f, t f^{\prime}, t^{2} f^{\prime \prime} \in \mathrm{L}^{2}(0, T ; V), f^{\prime}, t f^{\prime \prime} \in \mathrm{L}^{2}\left(0, T ; V^{*}\right)$. Then there is - if $d=3$ only for sufficiently small $T-$ a unique solution $u \in \mathcal{C}([0, T] ; \mathcal{D}(A))$ to Problem $(P)$ with $u^{\prime \prime}, t\left(f^{\prime \prime}-u^{\prime \prime \prime}\right) \in \mathrm{L}^{2}\left(0, T ; V^{*}\right), \sqrt{t} u^{\prime \prime}, t^{3 / 2}\left(f^{\prime \prime}-u^{\prime \prime \prime}\right) \in \mathrm{L}^{2}(0, T ; H)$ and $u^{\prime}, t u^{\prime \prime} \in \mathrm{L}^{2}(0, T ; V)$.

The proof of Theorem 1.1, which can be found in [2], follows from arguments given in [5, 10]. The results are optimal since higher, not time-weighted regularity is equivalent to global compatibility conditions. We set

$$
\begin{gathered}
K_{1}:=\max _{t \in[0, T]}\|u(t)\|, \quad K_{2}:=\max _{t \in[0, T]}|A u(t)|, \\
K_{3}:=\left\|u^{\prime}\right\|_{\mathrm{L}^{2}(0, T ; V)}^{2}, \quad K_{4}:=\left\|u^{\prime \prime}\right\|_{\mathrm{L}^{2}\left(0, T ; V^{*}\right)}^{2}, \quad K_{5}:=\left\|t\left(f^{\prime \prime}-u^{\prime \prime \prime}\right)\right\|_{\mathrm{L}^{2}\left(0, T ; V^{*}\right)}^{2} .
\end{gathered}
$$

For the time discretisation, let the time interval $[0, T]$ for given $N \in \mathbb{N}$ be equidistantly partitioned with the time step $\Delta t$ and $t_{n}:=n \Delta t(n=0, \ldots, N)$. We denote the backward divided differences by

$$
\mathrm{D}_{1} v^{n}:=\frac{v^{n}-v^{n-1}}{\Delta t}, \quad \mathrm{D}_{2} v^{n}:=\frac{3}{2} \mathrm{D}_{1} v^{n}-\frac{1}{2} \mathrm{D}_{1} v^{n-1}=\frac{1}{\Delta t}\left(\frac{3}{2} v^{n}-2 v^{n-1}+\frac{1}{2} v^{n-2}\right) .
$$

We also consider, for a Bochner integrable function $g$, the natural restrictions and the extrapolation

$$
\mathrm{R}_{1}^{n} g:=\frac{1}{\Delta t} \int_{t_{n-1}}^{t_{n}} g(t) \mathrm{d} t, \quad \mathrm{R}_{2}^{n} g:=\frac{3}{2} \mathrm{R}_{1}^{n} g-\frac{1}{2} \mathrm{R}_{1}^{n-1} g, \quad \mathrm{E} v^{n}:=2 v^{n-1}-v^{n-2} .
$$

Note that $\mathrm{R}_{q}^{n} v^{\prime}=\mathrm{D}_{q} v\left(t_{n}\right)=v^{\prime}\left(t_{n}\right)+\mathcal{O}\left((\Delta t)^{q}\right)(q \in\{1,2\})$ and $\mathrm{E} v\left(t_{n}\right)=v\left(t_{n}\right)+\mathcal{O}\left((\Delta t)^{2}\right)$ for smooth $v=v(t)$. The time discretisation of Problem $(\mathrm{P})$ for computing $u^{n}$ that approximates $u\left(t_{n}\right)$ then reads as

Problem $\left(\mathrm{P}_{\Delta t}\right)$. For given $u^{0}, u^{1} \in H$ and $f \in \mathrm{L}^{2}\left(0, T ; V^{*}\right)$, find $\left\{u^{n}\right\} \subset V$ such that for all $v \in V$

$$
\left(\mathrm{D}_{2} u^{n}, v\right)+\nu\left(\left(u^{n}, v\right)\right)+b\left(u^{n}, u^{n}, v\right)=\left\langle\mathrm{R}_{2}^{n} f, v\right\rangle, \quad n=2,3, \ldots, N .
$$

Besides, we consider (for $u^{0}, u^{1} \in V$ ) the linearized Problem $\left(\mathrm{LP}_{\Delta t}\right)$ with $b\left(u^{n}, u^{n}, v\right)$ being replaced by $b\left(\mathrm{E} u^{n}, u^{n}, v\right)$. In both problems, the starting values can be obtained by taking $u^{0}=u_{0}$ and computing $u^{1}$ from $u^{0}$ using the implicit Euler method. The use of $\mathrm{R}_{2}^{n} f$ instead of an arbitrary approximation $f^{n}$ is only for simplicity and avoids to consider the extra error $f^{n}-\mathrm{R}_{2}^{n} f$. As we have shown in [2], there is at least one solution to Problem $\left(\mathrm{P}_{\Delta t}\right)$ and a unique solution to Problem $\left(\mathrm{LP}_{\Delta t}\right)$. Furthermore, a solution to Problem $\left(\mathrm{P}_{\Delta t}\right)$ or $\left(\mathrm{LP}_{\Delta t}\right)$ is stable in $\mathrm{l}^{\infty}(0, T ; H)$ and $\mathrm{l}^{2}(0, T ; V)$, where $\mathrm{l}^{p}(S ; X)$ denotes the discrete counterpart of $\mathrm{L}^{p}(S ; X)$ for functions defined on a time grid. Finally, certain piecewise polynomial prolongations of $\left\{u^{n}\right\}$ converge towards a weak solution under quite general assumptions on the initial data and right-hand side as $\Delta t$ tends to 0 .

\section{Velocity error to Problem $\left(\mathrm{P}_{\Delta t}\right)$}

Let $e^{n}:=u\left(t_{n}\right)-u^{n}(n=0,1, \ldots, N)$ be the velocity error to Problem $\left(\mathrm{P}_{\Delta t}\right)$. The error equation

$\left(\mathrm{D}_{2} e^{n}, v\right)+\nu\left(\left(e^{n}, v\right)\right)+b\left(u\left(t_{n}\right), e^{n}, v\right)+b\left(e^{n}, u\left(t_{n}\right), v\right)-b\left(e^{n}, e^{n}, v\right)=\left\langle\rho^{n}, v\right\rangle \quad \forall v \in V, n=2,3, \ldots, N$ 
with the consistency error to the corresponding linear Stokes problem

$$
\begin{aligned}
\rho^{n} & =\mathrm{D}_{2} u\left(t_{n}\right)-u^{\prime}\left(t_{n}\right)+f\left(t_{n}\right)-\mathrm{R}_{2}^{n} f \\
& =\frac{1}{4 \Delta t}\left(\int_{t_{n-1}}^{t_{n}}\left(t_{n}-t\right)\left(t_{n}+3 t-4 t_{n-1}\right)\left(f^{\prime \prime}(t)-u^{\prime \prime \prime}(t)\right) \mathrm{d} t+\int_{t_{n-2}}^{t_{n-1}}\left(t-t_{n-2}\right)^{2}\left(f^{\prime \prime}(t)-u^{\prime \prime \prime}(t)\right) \mathrm{d} t\right)
\end{aligned}
$$

follows directly from (1) and (2).

In the sequel, let $C>0$ be a generic constant that may depend on the domain $\Omega$ and its dimension, on $T$, embedding constants, and $\nu$ but not on the exact solution, the initial data or right-hand side. Moreover, we set

$$
\|v\|:=\left(\max _{n=2, \ldots, N}\left|v^{n}\right|^{2}+(\Delta t)^{4} \sum_{j=1}^{N-1}\left|\mathrm{D}^{2} v^{j}\right|^{2}+\nu \Delta t \sum_{j=2}^{N}\left\|v^{j}\right\|^{2}\right)^{1 / 2}
$$

for a grid function $\left\{v^{j}\right\}$, where $\mathrm{D}^{2} v^{j}:=\left(v^{j+1}-2 v^{j}+v^{j-1}\right) /(\Delta t)^{2}$ denotes the second divided difference.

Theorem 2.1. Let $u \in \mathcal{C}([0, T] ; \mathcal{D}(A))$ and $t\left(f^{\prime \prime}-u^{\prime \prime \prime}\right) \in \mathrm{L}^{2}\left(0, T ; V^{*}\right)$. If $\Delta t$ is sufficiently small then

$$
\|e\|^{2} \leq \Lambda_{1}\left(\left|e^{0}\right|^{2}+\left|e^{1}\right|^{2}+K_{5}(\Delta t)^{2}\right), \quad \Lambda_{1}:=C \exp \left(\frac{C K_{2}^{4 / 3} T}{1-C K_{2}^{4 / 3} \Delta t}\right) .
$$

Proof. We set $v=e^{n}$ in (3) and observe $b\left(u\left(t_{n}\right), e^{n}, e^{n}\right)=b\left(e^{n}, e^{n}, e^{n}\right)=0$. With known estimates for $b(\cdot, \cdot, \cdot)$ (cf. [10]) and Young's inequality, we find

$$
\left|b\left(e^{n}, u\left(t_{n}\right), e^{n}\right)\right| \leq C\left|e^{n}\right|^{3 / 2}\left\|e^{n}\right\|^{1 / 2}\left|A u\left(t_{n}\right)\right| \leq C K_{2}^{4 / 3}\left|e^{n}\right|^{2}+\frac{\nu}{4}\left\|e^{n}\right\|^{2}, \quad\left|\left\langle\rho^{n}, e^{n}\right\rangle\right| \leq \nu^{-1}\left\|\rho^{n}\right\|_{*}^{2}+\frac{\nu}{4}\left\|e^{n}\right\|^{2} .
$$

The assertion follows with standard arguments from the identity

$$
4 \Delta t \sum_{j=2}^{n}\left(\mathrm{D}_{2} e^{j}, e^{j}\right)=\left|e^{n}\right|^{2}+\left|\mathrm{E} e^{n+1}\right|^{2}+(\Delta t)^{4} \sum_{j=1}^{n-1}\left|\mathrm{D}^{2} e^{j}\right|^{2}-\left|e^{1}\right|^{2}-\left|\mathrm{E} e^{2}\right|
$$

relation (4), and a discrete Gronwall lemma.

We remark that (5) follows from $4\left(\mathrm{D}_{2} v^{j}, v^{j}\right)=\mathrm{D}_{1}\left(\left|v^{j}\right|^{2}+\left|\mathrm{E} v^{j+1}\right|^{2}\right)+(\Delta t)^{3}\left|\mathrm{D}^{2} v^{j-1}\right|^{2}$ that reflects the G-stability of the two-step BDF. The theorem shows first-order convergence if $\left|e^{0}\right|,\left|e^{1}\right|=\mathcal{O}(\Delta t)$. It should be noted that optimal second order could be obtained from the estimate

$$
\Delta t \sum_{j=2}^{N}\left\|\rho^{j}\right\|_{*}^{2} \leq C(\Delta t)^{4}\left\|f^{\prime \prime}-u^{\prime \prime \prime}\right\|_{\mathrm{L}^{2}\left(0, T ; V^{*}\right)}^{2}
$$

However, the assumption $f^{\prime \prime}-u^{\prime \prime \prime} \in \mathrm{L}^{2}\left(0, T ; V^{*}\right)$ leads to global compatibility conditions. Since this seems to be inappropriate, we consider now the time-weighted error $\tilde{e}^{n}$. (We set $\tilde{v}^{n}:=t_{n} v^{n}$ for arbitrary $\left\{v^{n}\right\}$.)

Proposition 2.2. Under the assumptions of Theorem 2.1, it follows

$$
\|\tilde{e}\|^{2} \leq \Lambda_{1}\left((\Delta t)^{2}\left|e^{1}\right|^{2}+K_{5}(\Delta t)^{4}+\Delta t \sum_{j=2}^{N}\left\|\mathrm{E}^{j}\right\|_{*}^{2}\right) .
$$


Proof. Multiplying (3) by $t_{n}$ leads (because of $\widetilde{\mathrm{D}_{2} e^{n}}=\mathrm{D}_{2} \tilde{e}^{n}-\mathrm{E} e^{n}$ ) to

$$
\left(\mathrm{D}_{2} \tilde{e}^{n}, v\right)+\nu\left(\left(\tilde{e}^{n}, v\right)\right)+b\left(u\left(t_{n}\right), \tilde{e}^{n}, v\right)+b\left(\tilde{e}^{n}, u\left(t_{n}\right), v\right)-b\left(e^{n}, \tilde{e}^{n}, v\right)=\left\langle\tilde{\rho}^{n}, v\right\rangle+\left(\mathrm{E} e^{n}, v\right)
$$

and the proof is analogous to the proof of Theorem 2.1.

For proving a second-order estimate, it remains to show $\Delta t \sum_{j=2}^{N}\left\|\mathrm{E}^{j}\right\|_{*}^{2} \leq C(\Delta t)^{4}$. We shall employ a duality argument that is based upon a problem which can be interpreted as the backward-in-time, dual problem to a linearisation of Problem $\left(\mathrm{P}_{\Delta t}\right)$ by means of $u=\hat{u}+\delta u$ with "small" $\delta u$. So we consider for fixed $n \in\{2,3, \ldots, N\}$

Problem $\left(\mathrm{P}_{\Delta t, n}^{*}\right)$. For given $\phi^{n+1}=\phi^{n}=0$ and $g^{j}:=A^{-1} e^{j} \in V$ find $\phi^{j} \in V(j=n-1, \ldots, 0)$ such that

$$
\left(w, \mathrm{D}_{2}^{*} \phi^{j}\right)+\nu\left(\left(w, \phi^{j}\right)\right)+b\left(u\left(t_{j}\right), w, \phi^{j}\right)+b\left(w, u\left(t_{j}\right), \phi^{j}\right)=\left(w, g^{j}\right) \quad \forall w \in V
$$

Here $\mathrm{D}_{2}^{*} v^{j}:=\left(\frac{3}{2} v^{j}-2 v^{j+1}+\frac{1}{2} v^{j+2}\right) / \Delta t$ denotes the dual to the difference operator $\mathrm{D}_{2}$. Problem $\left(\mathrm{P}_{\Delta t, n}^{*}\right)$ admits a unique solution if $u \in \mathcal{C}([0, T] ; V)$ and $C K_{1}<\nu$. There is also a unique solution if $u \in \mathcal{C}([0, T] ; \mathcal{D}(A))$ and $\Delta t$ is sufficiently small (cf. [2]). In the following, let $\mathrm{E}^{*} v^{j}:=2 v^{j+1}-v^{j+2}$.

Proposition 2.3. Let $u \in \mathcal{C}([0, T] ; \mathcal{D}(A))$ and $u^{\prime} \in \mathrm{L}^{2}(0, T ; V)$. If $\Delta t$ is sufficiently small then

$$
\begin{gathered}
\Delta t \sum_{j=2}^{n-1}\left\|\mathrm{D}_{2}^{*} \phi^{j}\right\|^{2}+\max _{j=2, \ldots, n-1}\left(\left|A \phi^{j}\right|^{2}+\left|A \mathrm{E}^{*} \phi^{j-1}\right|^{2}\right) \leq \Lambda_{2} \Delta t \sum_{j=2}^{n-1}\left\|e^{j}\right\|_{*}^{2}, \quad n=3,4, \ldots, N, \\
\Lambda_{2}:=C\left(1+K_{1} K_{2}\right) \exp \left(\frac{C K_{1} K_{2} T+C \sqrt{K_{3} T}}{1-C K_{1} K_{2} \Delta t-C \sqrt{K_{3} \Delta t}}\right)
\end{gathered}
$$

holds. If $u^{\prime} \in \mathrm{L}^{2}(0, T ; V)$ and $u^{\prime \prime} \in \mathrm{L}^{2}\left(0, T ; V^{*}\right)$, it follows for arbitrary $\eta>0$ and $n=3,4, \ldots, N$

$$
\begin{gathered}
\Delta t \sum_{j=2}^{n-1}\left\|e^{j}\right\|_{*}^{2} \leq \eta\left(\Delta t \sum_{j=2}^{n-1}\left\|\mathrm{D}_{2}^{*} \phi^{j}\right\|^{2}+\max _{j=2, \ldots, n-1}\left(\left|A \phi^{j}\right|^{2}+\left|A \mathrm{E}^{*} \phi^{j-1}\right|^{2}\right)\right)+C \eta^{-1} R T, \\
R T=\left(\max _{j=2, \ldots, n-1}\left|e^{j}\right|^{2}\right) \Delta t \sum_{j=2}^{n-1}\left\|e^{j}\right\|^{2}+\left|A^{-1} e^{1}\right|^{2}+\left|A^{-1} \mathrm{E}^{2}\right|^{2}+(1+\eta)\left(K_{3}^{2}+K_{4}\right)(\Delta t)^{4} .
\end{gathered}
$$

Proof. We can only sketch the proof here and refer to [3] for more details. In a first step, we derive the estimate

$$
\max _{j=2, \ldots, n-1}\left(\left\|\phi^{j}\right\|^{2}+\left\|\mathrm{E}^{*} \phi^{j-1}\right\|^{2}\right)+(\Delta t)^{4} \sum_{j=3}^{n}\left\|\mathrm{D}^{2} \phi^{j}\right\|^{2}+\Delta t \sum_{j=2}^{n-1}\left|A \phi^{j}\right|^{2} \leq C\left(1-C K_{1} K_{2} \Delta t\right)^{2-n} \Delta t \sum_{j=2}^{n-1}\left\|e^{j}\right\|_{*}^{2} .
$$

Taking $w=A \mathrm{D}_{2}^{*} \phi^{j}$ in (7) and employing the identity

$$
\sum_{j=k}^{n-1}\left\|\mathrm{D}_{2} v^{j+2}\right\|^{2}=\sum_{j=k}^{n-1}\left\|\mathrm{D}_{2}^{*} v^{j}\right\|^{2}+2\left(\left\|\mathrm{D}_{1} v^{n+1}\right\|^{2}-\left\|\mathrm{D}_{1} v^{k+1}\right\|^{2}\right), \quad k=2,3, \ldots, n-1
$$

we can then prove the first assertion by applying a discrete Gronwall lemma. For the second part, the identity

$$
2 \sum_{j=2}^{n-1}\left(\left(\mathrm{D}_{2} v^{j}, w^{j}\right)-\left(v^{j}, \mathrm{D}_{2}^{*} w^{j}\right)\right)=\left(\mathrm{E} v^{n}, w^{n}\right)+\left(v^{n-1}, \mathrm{E}^{*} w^{n-1}\right)-\left(\mathrm{E} v^{2}, w^{2}\right)-\left(v^{1}, \mathrm{E}^{*} w^{1}\right) .
$$

plays an important rôle. 
Theorem 2.4. Let the assumptions of Theorem 2.1 and Proposition 2.3 be fulfilled. Then

$$
\|\tilde{e}\|^{2} \leq \Lambda_{1} \Lambda_{2}\left(e_{0}^{2}+r^{2}(\Delta t)^{4}\right)
$$

holds with $e_{0}^{2}:=\left|e^{0}\right|^{4}+\left|e^{1}\right|^{4}+(\Delta t)^{2}\left|e^{1}\right|^{2}+\Delta t\left(\left\|e^{0}\right\|_{*}^{2}+\left\|e^{1}\right\|_{*}^{2}\right)+\left|A^{-1} e^{0}\right|^{2}+\left|A^{-1} e^{1}\right|^{2}, r^{2}:=K_{3}^{2}+K_{4}+K_{5}\left(1+K_{5}\right)$.

Proof. The assertion follows immediately from Propositions 2.2 and 2.3 by taking $\eta=1 /\left(2 \Lambda_{2}\right)$, the first-order result of Theorem 2.1, and some tedious, but simple calculations.

It follows second-order convergence if $\left|A^{-s} e^{0}\right|,\left|A^{-s} e^{1}\right|=\mathcal{O}\left((\Delta t)^{1+s}\right)\left(s \in\left\{0, \frac{1}{2}, 1\right\}\right)$, where $\left|A^{-1 / 2} \cdot\right| \equiv\|\cdot\|_{*}$.

\section{Velocity error to Problem $\left(\mathrm{LP}_{\Delta t}\right)$}

For the velocity error to Problem $\left(\mathrm{LP}_{\Delta t}\right)$, we find with $\rho^{n}$ given by $(4)$ the error equation

$$
\begin{gathered}
\left(\mathrm{D}_{2} e^{n}, v\right)+\nu\left(\left(e^{n}, v\right)\right)+b\left(\mathrm{E} u\left(t_{n}\right), e^{n}, v\right)+b\left(\mathrm{E}^{n}, u\left(t_{n}\right), v\right)-b\left(\mathrm{E} e^{n}, e^{n}, v\right) \\
=\left\langle\rho^{n}, v\right\rangle-(\Delta t)^{2} b\left(\mathrm{D}^{2} u\left(t_{n-1}\right), u\left(t_{n}\right), v\right) \quad \forall v \in V, n=2,3, \ldots, N .
\end{gathered}
$$

Theorem 3.1. Let $u \in \mathcal{C}([0, T] ; \mathcal{D}(A)), \sqrt{t} u^{\prime \prime} \in \mathrm{L}^{2}(0, T ; H)$, and $t\left(f^{\prime \prime}-u^{\prime \prime \prime}\right) \in \mathrm{L}^{2}\left(0, T ; V^{*}\right)$. Then

$$
\|e\|^{2} \leq \Lambda_{3}\left(\left|e^{0}\right|^{2}+\left|e^{1}\right|^{2}+K_{5}(\Delta t)^{2}+K_{2}^{2}\left\|\sqrt{t} u^{\prime \prime}\right\|_{\mathrm{L}^{2}(0, T ; H)}^{2}(\Delta t)^{3}\right), \quad \Lambda_{3}:=C \exp \left(1+C K_{2}^{2} T\right) .
$$

Proof. The proof is rather similar to the proof of Theorem 2.1. We only have the additional term

$$
(\Delta t)^{2}\left|b\left(\mathrm{D}^{2} u\left(t_{n-1}\right), u\left(t_{n}\right), e^{n}\right)\right| \leq C(\Delta t)^{2}\left|\mathrm{D}^{2} u\left(t_{n-1}\right)\right|\left|A u\left(t_{n}\right)\right|\left\|e^{n}\right\| \leq C K_{2}^{2}(\Delta t)^{4}\left|\mathrm{D}^{2} u\left(t_{n-1}\right)\right|^{2}+\frac{\nu}{8}\left\|e^{n}\right\|^{2},
$$

for which we observe that $(\Delta t)^{5} \sum_{j=1}^{N-1}\left|\mathrm{D}^{2} u\left(t_{j}\right)\right|^{2} \leq C(\Delta t)^{3}\left\|\sqrt{t} u^{\prime \prime}\right\|_{\mathrm{L}^{2}(0, T ; H)}^{2}$.

The theorem shows first-order convergence if $\left|e^{0}\right|,\left|e^{1}\right|=\mathcal{O}(\Delta t)$. We emphasize that, as for an explicit scheme, there is no restriction on the time step size. With estimate (6) and

$$
(\Delta t)^{5} \sum_{j=1}^{N-1}\left|\mathrm{D}^{2} u\left(t_{j}\right)\right|^{2} \leq C(\Delta t)^{4}\left\|u^{\prime \prime}\right\|_{\mathrm{L}^{2}(0, T ; H)}^{2}
$$

we might have shown second order. Unfortunately, it seems to be inappropriate to assume $u^{\prime \prime} \in \mathrm{L}^{2}(0, T ; H)$.

We shall now consider the time-weighted error $\tilde{e}^{n}$, for which a result similar to Proposition 2.2 holds (cf. [3]). Also Proposition 2.3 remains valid (if in addition $t u^{\prime \prime} \in \mathrm{L}^{2}(0, T ; V)$ and $\sqrt{t} u^{\prime \prime} \in \mathrm{L}^{2}(0, T ; H)$ ) but with

$$
R T^{\prime}=R T+\left\|t u^{\prime \prime}\right\|_{\mathrm{L}^{2}(0, T ; V)}^{2}(\Delta t)^{3} \sum_{j=2}^{n-1}\left\|e^{j}\right\|^{2}+K_{2}^{2}(\Delta t)^{5} \sum_{j=1}^{n-2}\left|\mathrm{D}^{2} e^{j}\right|^{2}+K_{2}^{2}(\Delta t)^{3}\left\|\sqrt{t} u^{\prime \prime}\right\|_{\mathrm{L}^{2}(0, T ; H)}^{2}
$$

instead of $R T$ from (8). Obviously, the last two terms are of suboptimal order $3 / 2$. For better results, we would need an estimate of the type $|b(u, v, w)| \leq C\|u\|_{*}|A v||A w|$, which is not at hand. So we loose half an order in $\Delta t$.

Theorem 3.2. Under the regularity asserted in Theorem 1.1 and if $\Delta t$ is sufficiently small, the estimate

$$
\|\tilde{e}\|^{2} \leq \Lambda_{2} \Lambda_{3}\left(e_{0}^{2}+K_{2}^{2}\left(\left|e^{0}\right|^{2}+\left|e^{1}\right|^{2}\right) \Delta t+r_{1}^{2}(\Delta t)^{3}+\left(r^{2}+r_{2}^{2}\right)(\Delta t)^{4}\right)
$$


holds with $e_{0}$ and $r$ as in Theorem 2.4, $r_{1}:=K_{2}^{2}\left(K_{5}+\left\|\sqrt{t} u^{\prime \prime}\right\|_{L^{2}(0, T ; H)}^{2}\right)$ and

$$
r_{2}:=K_{2}^{2}\left(\left\|t u^{\prime \prime}\right\|_{\mathrm{L}^{2}(0, T ; H)}^{2}+K_{2}^{2}\left\|\sqrt{t} u^{\prime \prime}\right\|_{\mathrm{L}^{2}(0, T ; H)}^{2}+K_{2}^{2}\left\|\sqrt{t} u^{\prime \prime}\right\|_{\mathrm{L}^{2}(0, T ; H)}^{4}(\Delta t)^{2}\right)+\left\|t u^{\prime \prime}\right\|_{\mathrm{L}^{2}(0, T ; V)}^{4} .
$$

Proof. The assertion follows as in the proof of Theorem 2.4 by taking $\eta=1 /\left(2 \Lambda_{2}\right)$.

\section{Reintroduction of the pressure}

After the velocity field $\left\{u^{n}\right\}$ is determined, we may compute approximations $p^{n}(n=2,3, \ldots, N)$ for the pressure $p\left(t_{n}\right)$ from the variational formulation in the function spaces $\mathrm{H}_{0}^{1}(\Omega)^{d} \ni u(t)$ and $\mathrm{L}^{2}(\Omega) / \mathbb{R} \ni p(t)$ :

$$
\left(p^{n}, \nabla \cdot v\right)=\left(\mathrm{D}_{2} u^{n}, v\right)+\nu\left(\left(u^{n}, v\right)\right)+b\left(u^{n}, u^{n}, v\right)-\left\langle\mathrm{R}_{2}^{n} f, v\right\rangle \quad \forall v \in \mathrm{H}_{0}^{1}(\Omega)^{d} .
$$

For the error $\pi^{n}:=p\left(t_{n}\right)-p^{n}(n=2,3, \ldots, N)$, it follows the error equation

$$
\left(\pi^{n}, \nabla \cdot v\right)=\left(\mathrm{D}_{2} e^{n}, v\right)+\nu\left(\left(e^{n}, v\right)\right)+b\left(u\left(t_{n}\right), e^{n}, v\right)+b\left(e^{n}, u\left(t_{n}\right), v\right)-b\left(e^{n}, e^{n}, v\right)-\left\langle\rho^{n}, v\right\rangle \quad \forall v \in \mathrm{H}_{0}^{1}(\Omega)^{d} .
$$

From Babuška-Brezzi's condition (cf. [4]),

$$
\exists \ell>0 \forall q \in \mathrm{L}^{2}(\Omega) / \mathbb{R}: \sup _{v \in \mathrm{H}_{0}^{1}(\Omega)^{d} \backslash\{0\}} \frac{(q, \nabla \cdot v)}{\|v\|} \geq \ell\|q\|_{\mathrm{L}^{2}(\Omega) / \mathbb{R}}
$$

with $\|q\|_{\mathrm{L}^{2}(\Omega) / \mathbb{R}}:=\inf _{c \in \mathbb{R}}\|q+c\|_{\mathrm{L}^{2}(\Omega)}$, we conclude

$$
\left\|\pi^{n}\right\|_{\mathrm{L}^{2}(\Omega) / \mathbb{R}} \leq C \ell^{-1}\left(\left\|\mathrm{D}_{2} e^{n}\right\|_{-1}+\left\|e^{n}\right\|+K_{1}\left\|e^{n}\right\|+\left|e^{n}\right|^{1 / 2}\left\|e^{n}\right\|^{3 / 2}+\left\|\rho^{n}\right\|_{-1}\right),
$$

where $\|\cdot\|_{-1}$ denotes the $\mathrm{H}^{-1}(\Omega)^{d}$-norm. Note that $V \subset \mathrm{H}_{0}^{1}(\Omega)^{d}$ implies $\|g\|_{-1} \geq\|g\|_{*}$ for all $g \in \mathrm{H}^{-1}(\Omega)^{d} \subset V^{*}$.

Theorem 4.1. Let $u \in \mathcal{C}([0, T] ; V)$ and $t^{3 / 2}\left(f^{\prime \prime}-u^{\prime \prime \prime}\right) \in \mathrm{L}^{2}(0, T ; H)$. Suppose further for some $q>0$ that

$$
\max _{n=0, \ldots, N}\left|e^{n}\right|^{2}+\Delta t \sum_{j=0}^{N}\left\|e^{j}\right\|^{2} \leq C(\Delta t)^{2}, \quad \max _{n=0, \ldots, N}\left|\tilde{e}^{n}\right|^{2}+\Delta t \sum_{j=0}^{N}\left\|\tilde{e}^{j}\right\|^{2} \leq C(\Delta t)^{2(1+q)} .
$$

Then $\left\|\tilde{\pi}^{n}\right\|_{\mathrm{L}^{2}(\Omega) / \mathbb{R}}(n=2,3, \ldots, N)$ is of order $\mathcal{O}\left((\Delta t)^{\min (q, 1)}\right)$.

Proof. Multiplying (9) by $t_{n}$ leads to

$$
\left\|\tilde{\pi}^{n}\right\|_{\mathrm{L}^{2}(\Omega) / \mathbb{R}} \leq C \ell^{-1}\left(\left\|\mathrm{D}_{2} \tilde{e}^{n}\right\|_{-1}+\left\|\mathrm{E}^{n}\right\|_{-1}+\left\|\tilde{e}^{n}\right\|+K_{1}\left\|\tilde{e}^{n}\right\|+\left|e^{n}\right|^{1 / 2}\left\|e^{n}\right\|^{1 / 2}\left\|\tilde{e}^{n}\right\|+\left\|\tilde{\rho}^{n}\right\|_{-1}\right) .
$$

In view of the continuous embedding $\mathrm{L}^{2}(\Omega)^{d} \hookrightarrow \mathrm{H}^{-1}(\Omega)^{d}$, we have with (10)

$$
\left\|\mathrm{D}_{2} \tilde{e}^{n}\right\|_{-1} \leq C(\Delta t)^{q}, \quad\left\|\mathrm{E} e^{n}\right\|_{-1} \leq C \Delta t, \quad\left\|\tilde{e}^{n}\right\| \leq C(\Delta t)^{1 / 2+q}, \quad\left|e^{n}\right|^{1 / 2}\left\|e^{n}\right\|^{1 / 2}\left\|\tilde{e}^{n}\right\| \leq C(\Delta t)^{5 / 4+q} .
$$

We finally obtain from (4) with standard arguments $\left\|\tilde{\rho}^{n}\right\|_{-1} \leq C\left|\tilde{\rho}^{n}\right| \leq C \Delta t\left\|t^{3 / 2}\left(f^{\prime \prime}-u^{\prime \prime \prime}\right)\right\|_{L^{2}(0, T ; H)}$.

So $t_{n} \pi^{n}$ is of order 1 for the nonlinear $(q=1)$ and of order $1 / 2$ for the linearized method $(q=1 / 2)$. A similar first-order estimate of $t_{n}^{3 / 2} \pi^{n}$ has been presented in [5] for the Crank-Nicolson scheme. 


\section{REFERENCES}

[1] G.A. Baker, V.A. Dougalis and O.A. Karakashian, On a higher order accurate fully discrete Galerkin approximation to the Navier-Stokes equations. Math. Comp. 39 (1982) 339-375.

[2] E. Emmrich, Analysis von Zeitdiskretisierungen des inkompressiblen Navier-Stokes-Problems. Cuvillier, Göttingen (2001).

[3] E. Emmrich, Error of the two-step BDF for the incompressible Navier-Stokes problem. Preprint 741, TU Berlin (2002).

[4] V. Girault and P.-A. Raviart, Finite Element Approximation of the Navier-Stokes Equations. Springer, Berlin (1979).

[5] J.G. Heywood and R. Rannacher, Finite element approximation of the nonstationary Navier-Stokes problem, Part IV: Error analysis for second-order time discretization. SIAM J. Numer. Anal. 27 (1990) 353-384.

[6] A.T. Hill and E. Süli, Approximation of the global attractor for the incompressible Navier-Stokes equations. IMA J. Numer. Anal. 20 (2000) 633-667.

[7] S. Müller-Urbaniak, Eine Analyse des Zwischenschritt- $\theta$-Verfahrens zur Lösung der instationären Navier-Stokes-Gleichungen. Preprint 94-01 (SFB 359), Univ. Heidelberg (1994).

[8] A. Prohl, Projection and Quasi-compressibility Methods for Solving the Incompressible Navier-Stokes Equations. Teubner, Stuttgart (1997).

[9] R. Temam, Navier-Stokes Equations. Theory and Numerical Analysis. North-Holland Publ. Company, Amsterdam (1977).

[10] R. Temam, Navier-Stokes Equations and Nonlinear Functional Analysis. CBMS-NSF Reg. Confer. Ser. Appl. Math. SIAM 41 (1985).

To access this journal online:

www.edpsciences.org 